\title{
Investigating the Specificity of Peptide Adsorption on Gold Using Molecular Dynamics Simulations
}

\author{
Ana Vila Verde, ${ }^{\dagger}$ Jacqueline M. Acres, and Janna K. Maranas* \\ The Pennsylvania State University, Department of Chemical Engineering, \\ University Park, Pennsylvania 16802
}

Received February 26, 2009; Revised Manuscript Received May 26, 2009

\begin{abstract}
We report all-atom molecular dynamics simulations following adsorption of gold-binding and non-gold-binding peptides on gold surfaces modeled with dispersive interactions. We examine the dependence of adsorption on both identity of the amino acids and mobility of the peptides. Within the limitations of the approach, results indicate that when the peptides are solvated, adsorption requires both configurational changes and local flexibility of individual amino acids. This is achieved when peptides consist mostly of random coils or when their secondary structural motifs (helices, sheets) are short and connected by flexible hinges. In the absence of solvent, only affinity for the surface is required: mobility is not important. In combination, these results suggest the barrier to adsorption presented by displacement of water molecules requires conformational sampling enabled through mobility.
\end{abstract}

\section{Introduction}

Many natural materials are organic/inorganic nanocomposites with remarkable properties. For example, mother-of-pearl is a brittle ceramic material held together by small amounts of protein: this nanocomposite resists permanent deformation and fracture more than its individual components. Equally remarkable is the fact that organic/inorganic nanocomposites selfassemble at ambient conditions. Self-assembly is often a twostep process in which the organic fraction drives precipitation of the inorganic fraction from solution to form nanoparticles, ${ }^{1-3}$ and then directs the self-organization of these nanoparticles to form larger structures. Precipitation and self-organization are enabled by strong and specific organic-inorganic interactions, which we term high affinity. In high affinity organic-inorganic pairs, "strong" means that the free energy of adsorption approaches or exceeds $10 \mathrm{kcal} / \mathrm{mol}$ and "specific" means that the organic molecules adsorb on the inorganic surface of interest but not on others. Proteins show high affinity for inorganic materials in many natural processes, and as in the mother-ofpearl example above, often make up the organic fraction of natural organic/inorganic nanocomposites.

Natural nanocomposites have inspired searches for artificial counterparts with tailored mechanical, electrical, magnetic, or optical properties, including new methods of producing inorganic nanoscale blocks at ambient conditions [precipitation] and the formation of these building blocks into useful structures [self-organization]. Producing artificial nanocomposites requires identification of proteins, typically small peptides, with high affinities for the inorganics important from a nanotechnology standpoint. Several high-throughput methods like cell or phage display have been used to test large peptide libraries for affinity to specific inorganics. Artificial peptides now exist that target gold ${ }^{4,5}$ chromium, ${ }^{5}$ titanium, ${ }^{6}$ cobalt-platinum hybrids, ${ }^{7}$ silver, ${ }^{8}$ and several insulator and oxide surfaces. ${ }^{9,10}$ Despite this progress, a design-oriented approach for protein-inorganic

* To whom correspondence should be addressed. Tel.: +1 (814) 8636228. Fax: +1 (814) 865-7846. E-mail: jmaranas@psu.edu.

Current address: Universidade do Minho, Departamento de Física, Campus de Gualtar, 4710-057 Braga, Portugal. adsorption remains limited because we do not fully understand the reasons driving high affinity protein-inorganic adsorption. To enable a design-oriented approach, we require a theoretical framework that explains experimental observations and provides guidelines for selecting high affinity protein-inorganic pairs. Important for this framework is a molecular-level description of peptide adsorption that identifies and isolates contributing factors: examples include amino acid identity and associated characteristics (charge and polarity), sequence, protein flexibility, and structural stability in solution.

The influence of amino acid identity and associated characteristics on protein adsorption to metals, insulators, and metaloxide surfaces has been addressed experimentally. To isolate amino acid identity, small homopolypeptides made from amino acids with varying polarity and charge were adsorbed on $\mathrm{Al}$, $\mathrm{Pt}, \mathrm{Ti}, \mathrm{Au}, \mathrm{Pd}, \mathrm{GaAs}, \mathrm{Si}_{3} \mathrm{~N}_{4}, \mathrm{SiO}_{2}$, and $\mathrm{AlGaAs}$ surfaces, and the resulting surface densities were characterized..$^{11}$ The authors found that electrostatics drive adsorption to insulator or oxide surfaces but observed no clear pattern for metals. For example, on gold surfaces, homopolypeptides with similar characteristics behaved differently: polylysine [polar and charged] did not adsorb to gold, whereas polyarginine [also polar and charged] did. This suggests that something beyond amino acid characteristics controls adsorption to metals. Although there are no common characteristics, six amino acids adsorb strongly to gold as homopolypeptides: arginine (denoted by $\mathrm{R}$ in one letter code for amino acids) and aspartic acid (D) are polar and charged; serine $(\mathrm{S})$, threonine $(\mathrm{T})$, and proline $(\mathrm{P})$ are polar and uncharged; and isoleucine (I) is apolar. The importance of serine and threonine in gold adsorption has also been observed in simulations. ${ }^{12}$

It is possible that, although the characteristics of individual amino acids do not control adsorption, the arrangement of those amino acids, that is, peptide sequence, is important. For example, energy minimization of five heptapeptides in vacuum revealed that the peptides adopt a configuration that matches the surface morphology. ${ }^{13}$ The peptides selected for study ranged from weakly to strongly adsorbing, with little correlation between the number of high affinity amino acids identified by Willett ${ }^{11}$ 
and adsorption behavior. The importance of sequence arises from the observation that the preferred configurations of strongly adsorbing sequences allow high affinity amino acids to interact strongly with the surface. In contrast, high affinity amino acids in configurations of weakly adsorbing sequences are located further from the surface and cannot contribute to binding. Thus, the presence of high affinity amino acids is not sufficient; arrangement of those amino acids such that they may interact with the surface is also required.

Protein flexibility and stability in solution are related characteristics. ${ }^{14-16}$ Stability refers to the ability of the protein to maintain its overall conformation, whereas protein flexibility refers to oscillations about this overall conformation. Some studies address the relevance of peptide flexibility for adsorption on $\mathrm{Au}$ and Pt. The gold-binding peptide 3GBP, studied in solution using circular dichroism and NMR spectroscopy, exists mainly as a random coil and is, thus, more flexible than peptides with a well-defined folded structure. ${ }^{17}$ This suggests that the ability of a protein to explore a variety of conformations surrounding its stable structure is relevant to adsorption. For platinum, molecular dynamics simulations of strong and weak Pt-binding peptides indicate that strong binders frequently contain the structural motif TST. ${ }^{18}$ Although both threonine and serine bind strongly to gold surfaces as homopolypeptides, neither shows a similar affinity for platinum. ${ }^{11}$ Because TST is the most flexible region of the peptides in their study and appears frequently in Pt-binding peptides, the authors suggested that the motif's flexibility is important for the peptide's affinity for platinum. The link between peptide adsorption and structural stability was introduced with simulations of model systems consisting of small chains and generic surfaces. ${ }^{19}$ The chains capture protein-like behavior [i.e., they fold into a unique compact structure at the global energy minimum] by incorporating two types of beads (hydrophobic and polar). The results show that if the protein stability is sufficiently small [i.e., its overall conformation exhibits large fluctuations] adsorption can occur even when amino acid/surface interactions are weak. Results also show that even highly stable proteins adsorb strongly on surfaces when the protein-surface interactions are strong or when the particular protein structure allows it. Although we now recognize some of the generic trends governing adsorption, we are not yet able to predict how strongly proteins [characterized by their sequence, structure flexibility and stability] will adsorb on a specific surface.

Our work addresses flexibility and stability of protein conformation and its impact on adsorption on metals, via atomistic molecular dynamics simulation. We select gold as the metallic surface because peptides with a range of affinities for gold have been characterized. ${ }^{5,11} \mathrm{We}$ are thus able to select two categories of peptides: gold-binding, and non-gold-binding. Each peptide contains a 14 amino acid sequence: the gold-binding peptide (GBP) [sequence MHGKTQATSGTIQS, high affinity], and the non-gold-binding peptide (NGBP) [sequence AIRRDVNCIGASMH, low affinity]. ${ }^{5}$ We choose these sequences because both contain six amino acids with high affinity for gold as homopolypeptides, ${ }^{11}$ yet opposite behavior is observed. We highlight the amino acids with high affinity for gold using bold text. The different binding behavior illustrates that amino acid identity is not the dominant factor for adsorption of GPB and NGBP to gold, and thus other factors such as the ones we examine may play a role. We consider two sizes in our study: six-repeat peptides of both sequences (6GBP, 6NGBP), and a three-repeat peptide of the gold-binding sequence (3GBP). The non-gold-binding three repeat sequence 3 NGBP was not stud- ied because its behavior has not been determined experimentally. Of the peptides we consider, 3GBP has received the most attention: it adsorbs strongly to gold surfaces (adsorption free energy $\approx 8 \mathrm{kcal} / \mathrm{mol}$ ), shows specificity for gold over other substrates (e.g., $\mathrm{Pt}, \mathrm{SiO}_{2}$ ), and drives precipitation of gold from solution. $^{17,20,21}$

To investigate a potential link between the flexibility and stability of gold-binding peptides and their adsorption characteristics, we perform simulations in three circumstances: peptides in solution, solvated peptides approaching a gold surface, and peptides approaching a gold surface in vacuum. The solution state is the relevant system in which to assess flexibility and stability of GBP and NGBP, as these features allow the peptide to achieve the most favorable conformation for presentation to the surface. We examine adsorption by introducing the gold surface, which we expect to reduce flexibility and stability once peptide-surface interactions are established. Due to their limited duration, simulations cannot be expected to describe the entire process of adsorption: this process occurs over time scales outside of computational limitations, and includes movement of peptides to a concentrated region extending hundreds of nanometers from the protein surface. Instead, we follow the last stages of adsorption and link the behavior observed during this stage to their flexibility and stability in solution.

\section{Experimental Section}

As described above, our investigation uses molecular dynamics simulations to examine GBP and NGBP, in explicit solvent and on gold surfaces in solvated and vacuum conditions. All simulations are performed using the CHARMM22 force field for the peptides and buffering ions and the modified TIP3P (mTIP3P) model for water. ${ }^{22,23}$ We do not vary the $\mathrm{pH}$, considering all systems at a neutral $\mathrm{pH}$ of 7 . Most force fields, CHARMM22 included, do not contain parameters for elemental metals. As a result, we must choose the level of detail with which to model gold, and obtain the required parameters from the literature or by imposing agreement with appropriate data. For computational modeling of metals, tight binding, density functional and other quantum-mechanical methods are most accurate. ${ }^{24,25}$ Semiempirical embedded atom models are less costly and more accurate, ${ }^{26}$ but merging them with the force fields normally used to model biological and organic molecules is difficult. As a result, we choose to model the gold surface at the classical level, using the Lennard-Jones $[\mathrm{LJ}]$ potential. This carries the assumption that water and peptides physisorb, ignoring the possibility of chemisorption. While this is consistent with adsorption energies of water on gold, ${ }^{25}$ it is reasonable to question whether some amino acids [in our case cysteine and methionine] may chemisorb. Cysteine is only found in the non-goldbinding peptide, and thus we expect that covalent bonding does not occur. Experimental literature is not conclusive with respect to the ability of methionine to form covalent bonds, but because the goldbinding peptides in our study desorb in the presence of a surfactant, ${ }^{5}$ it is unlikely this occurs here. We also note that methionine does not show affinity to gold as a homopolymer, limiting the possibility of chemisorption. ${ }^{11}$ We assume that the gold atoms are unreactive to the water and peptide surrounding them by not advancing their positions in time and ignoring polarization of the metal. While the assumption that the gold atoms do not move in response to surface interactions is reasonable, neglecting polarization is potentially more serious. Studies of water on metal surfaces with and without polarization indicate that it does not significantly influence interfacial water structure, ${ }^{27}$ the energy from polarization is less than $10 \%$ of the total energy, ${ }^{28}$ and the interaction of charges with their images lead to cancellation, diminishing the influence of polarizability. ${ }^{29,30}$ Given the computational advantage of not advancing the gold atoms in time, and the comparable physical nature of the interactions [CHARMM does not include polarizability 
of water or peptide], we accept the limitations of our model. We cannot make predictions regarding the local binding or ordering near the gold surface and instead describe the relative interactions of the surface with water and the peptides. The former and other related issues require more realistic and detailed descriptions of surface-surface and surface-water interactions and are in themselves an active area of research. ${ }^{31,32}$ Our chosen approach reduces the required parameters to nonbonded LJ parameters $\sigma$ and $\varepsilon$ for gold. Interactions with water and peptide are then determined by the $\mathrm{LJ}$ parameters for the relevant atoms using combining rules. CHARMM22 uses a 6-12 LJ potential for which parameters have not yet appeared in the literature. We assign the parameters based on the interaction of water with gold, requiring both zero contact angle and agreement with single molecule ab initio adsorption energies. A similar approach was used to assign parameters for the 10-4 LJ potential. ${ }^{31}$ The parametrization is described in the Supporting Information. The resulting parameters, $\sigma_{\mathrm{Au}, \mathrm{Au}} / 2=1.6465$ $\AA$ and $\varepsilon_{\mathrm{Au}, \mathrm{Au}}=1.05 \mathrm{kcal} / \mathrm{mol}$, differ from those of ref 31 and another study $^{33}$ reporting 6-12 LJ potentials that appeared after our original submission because the mTIP3P potential for water has LJ interactions with both the oxygen and hydrogen atoms in water when implemented in CHARMM, whereas most models for water include only the oxygen. Because the $\mathrm{LJ}$ potential is short ranged, we simulate $\mathrm{Au}(001)$ or $\mathrm{Au}(111)$ surfaces using four atomic layers.

We use the simulation package NAMD, Scalable Molecular Dynamics, ${ }^{34}$ and the visualization package VMD, Visual Molecular Dynamics. ${ }^{35}$ Simulations are performed in either the NPT or the NVT ensembles at $310 \mathrm{~K}$ and a pressure of one atmosphere. To impose the desired temperatures, we use an Andersen thermostat during equilibration and Langevin dynamics during production runs. In the NPT ensemble, the pressure is maintained using a modified Nosé-Hoover barostat with Langevin dynamics to control fluctuations. van der Waals interactions are truncated at $12 \AA$, with a smoothing function applied between 10 and $12 \AA$. Electrostatic interactions are calculated directly up to distances of $12 \AA$; for longer distances, the Particle Mesh Ewald method with a grid spacing of $1 \AA$ is used. Integration is done using a modified Verlet algorithm. We use 1 fs time-steps; bonded forces are calculated at every time-step and van der Waals forces and electrostatics are calculated every two and four steps, respectively. All simulations use parallelepiped simulation boxes with periodic boundary conditions. We select the box size so that the protein and its periodic images are separated by at least $12 \AA$ and the protein does not interact with its periodic images via van der Waals potentials.

To obtain starting configurations for MD simulations, we require the solution structures of $3 \mathrm{GBP}, 6 \mathrm{GBP}$, and $6 \mathrm{NGBP}$. One limitation of our study is that structures of these peptides are not available in the protein data bank. We obtain structural estimates by combining publicly available servers for structure prediction [Robetta, JPRED, NNpredict and PredictProtein], ${ }^{36-39}$ available experimental information, and MD simulations in water. Selection of the most stable structure for each peptide is described in the Results and Discussion section. These structures are used as starting points for all MD simulations. For simulations with gold surfaces, the peptide is placed at the XY center of the surface, with the peptide atoms at least $5 \AA$ from the gold. We solvate each peptide using the equilibrated water box feature from VMD and $0.1 \mathrm{M} \mathrm{KCl}$. We increase the criteria for minimum box size $[X Y$ dimensions] from 12 to $17 \AA$ of water between images to account for conformational changes that may increase the size of the protein. Ideally the $Z$ dimension is selected such that bulk water is recovered between the peptide and the gold surface. This requires that the water layer extends $6 \AA$ from the protein ${ }^{40}$ and $7 \AA$ (see Figures S. 3 and S.4) from the gold surface, resulting in a minimum of $13 \AA$ between the peptide and the gold. Although this was feasible for the 3-repeat peptides, the required system size for the 6-repeat peptides caused us to relax this slightly: we instead used a minimum of $10 \AA$ between protein and gold. While not ideal, this distance still prevents contact between the first solvation shells of protein and gold; only the second solvation shells,
Table 1. System Sizes for MD Simulations of Solvated Peptides on $\mathrm{Au}(001)$

\begin{tabular}{lcccc}
\hline & \multicolumn{3}{c}{ minimum thickness of water, $\AA$} & \\
\cline { 2 - 4 } peptide & $\begin{array}{c}\text { any direction } \\
\text { in } X Y \text { plane }\end{array}$ & $\begin{array}{c}\text { above } \\
\text { protein }\end{array}$ & $\begin{array}{c}\text { below } \\
\text { protein }\end{array}$ & $\begin{array}{c}\text { number of } \\
\text { atoms }\end{array}$ \\
\hline 3GBP & 17 & 22 & 15 & 30947 \\
6GBP & 20 & 14 & 10 & 37879 \\
6NGBP & 20 & 15 & 10 & 38641 \\
\hline
\end{tabular}

Table 2. System Sizes for MD Simulations of Peptides on $\mathrm{Au}(001)$ in Vacuum

\begin{tabular}{lcc}
\hline \multicolumn{1}{c}{ peptide } & cell dimensions, $\AA^{3}$ & number of atoms \\
\hline 3GBP & $100 \times 100 \times 60$ & 5594 \\
6GBP6185 & & 6269 \\
6NGBP & & 6 \\
\hline
\end{tabular}

which behave more closely to bulk water, may interact. We present simulation cell sizes and the resulting number of atoms in Tables 1 and 2.

The initial systems as described above (most stable protein structure + equilibrated water box, most stable protein structure + gold surface + equilibrated water box) are equilibrated as follows. Solvated peptides without gold are run in the NPT ensemble at $1 \mathrm{~atm}$ and $310 \mathrm{~K}$ for 3 ns. This duration is sufficient to equilibrate protein-water contacts. ${ }^{40}$ Solvated peptides with gold are equilibrated for a total of $2.5 \mathrm{~ns}: 0.5$ ns in the NPT ensemble (peptide and water only), 1 ns in the NPT ensemble at $1 \mathrm{~atm}$ (full system), and $1 \mathrm{~ns}$ in the NVT ensemble using the volume returned from the NPT simulations. This time period exceeds the time required for the slowest water molecules (those near the gold surface) to reach the diffusive regime [see Figure S.4]. Production runs for proteins in solution are $5 \mathrm{~ns}$. Adsorption simulations are run for $18 \mathrm{~ns}$ in vacuum and $30-40 \mathrm{~ns}$ in solution. This time period allows the proteins to reach an adsorbed conformation that remains stable for $5-10 \mathrm{~ns}$.

\section{Results and Discussion}

Assessing the role of flexibility and stability in adsorption of proteins to gold surfaces required four steps, each of which are discussed below. As mentioned in the Experimental Section, our investigation required the prediction of 3GBP, 6GBP, and 6NGBP solution structures. Because these are not available in the Protein Data Bank, we describe how these structures were selected. We then consider adsorption of these proteins to gold surfaces in vacuum. The absence of water removes the hydrophobic effect which may act to stabilize the protein. As described below, the non-gold-binding peptide 6NGBP is more structured when solvated than its counterpart 6GBP. If stability and flexibility play a role, removing this difference should impact adsorption. We next consider adsorption for solvated systems, and close by considering the behavior of the peptides in solution and asking if this correlates with the observed variations in adsorption.

Predicting the Structure of 3GBP, 6GBP, and 6NGBP. For each peptide, the structure predictor program Robetta generates several possible structures; these differ in the location and extent of secondary structural motifs. The structures generated have $40 \%$ [3GBP] and 25\% [6GBP] of amino acids in small sheets or helices, with the remainder random coil. The structures of 6NGBP are dominated by five helices that include $80 \%$ of amino acids. All structures are consistent with the output from secondary structure predictors JPRED, NNpredict, and PredictProtein. ${ }^{37-39}$ To choose among the possible structures provided by Robetta, we simulate each of them in water and 
assess their relative stabilities by raising the temperature from $310 \mathrm{~K}$ in $10 \mathrm{~K}$ increments. We monitor the root-mean-square displacements (RMSD) of the whole protein and of individual helices and sheets, neglecting rotation and translation:

$$
\operatorname{RMSD}=\sqrt{\frac{\sum_{i=1}^{N}\left(\vec{r}_{t}-\vec{r}_{t=0}\right)^{2}}{N}}
$$

Atomic coordinates at times $t$ and 0 after eliminating rotation and translation are denoted by $\vec{r}_{t}$ and $\vec{r}_{t=0}$, and $N$ is the total number of atoms.

As an example, consider 6GBP. In this case, Robetta generates three structures, which we denote by $6 \mathrm{GBPa}, 6 \mathrm{GBPb}$, and $6 \mathrm{GBPc}$ (images not shown). Both $6 \mathrm{GBPa}$ and $6 \mathrm{GBPb}$ have five helices and 6GBPc has six. We simulate each structure for three ns blocks, raising the temperature by $10 \mathrm{~K}$. During simulation at 310 and $320 \mathrm{~K}$, the RMSD for all three structures is similar. However, two helices in 6GBPc have disappeared, indicating that this structure is less stable than the other two. Simulating the remaining structures for another three ns block at $320 \mathrm{~K}$ followed by $330 \mathrm{~K}$ confirms that $6 \mathrm{GBPb}$ is the most stable structure. We used a similar procedure to identify the most stable structures for $3 \mathrm{GBP}$ and $6 \mathrm{NGBP}$. The selected structures for 6GBP and 6NGBP contain 30 and $70 \%$ of amino acids in helices, respectively, after 5 ns simulations in water at $310 \mathrm{~K}$. Under the same conditions, the selected structure for 3 GBP contains $30 \%$ of its amino acids in two small $\beta$-sheets. This analysis shows a difference in structure between the goldbinding and the non-gold-binding peptides: the non-gold-binding peptide is highly structured, whereas 6GBP and 3GBP are relatively unstructured. Unfortunately, we cannot verify this difference against experiment. Some verification is available for 3GBP: NMR results indicate that this peptide takes a randomcoil-extended structure conformation, ${ }^{17}$ consistent with our prediction. It is thus reasonable to assume that our structures for the gold-binding peptides are representative. Although it is unlikely that $6 \mathrm{NGBP}$ is more structured than these predictions, it might be less structured. We consider the implications of this later in the paper.

Simulations of Peptides on Gold in Vacuum. Adsorption depends on the orientation of the peptide relative to the gold surface, yet computational limitations prevent significant rotation of the peptide during the simulation time. To overcome this difficulty, we consider adsorption starting from 10 different orientations relative to the gold surface. These orientations are meant to be representative of rotational sampling.

The potential energy of all systems decreases with increased adsorption, as illustrated in Figure 1. To determine the fraction of adsorbed atoms, we consider an atom adsorbed if it has displaced the second water layer from the gold surface. The first and second layers present sharp peaks in the density [see Figure S.3 in the Supporting Information and ref 32], divided by a minimum at $\sim 5 \AA$ from the surface. We thus consider an atom adsorbed if it is within $5 \AA$ of the gold surface. We conclude that, for all three peptides, interactions with the surface are favorable in vacuum. The final adsorbed configuration depends on orientation, with the fraction of adsorbed atoms varying from 15 to $28 \%$ for $6 \mathrm{GBP}$ and comparable variations observed for the other peptides. We take the lowest energy configurations as those most likely to correspond to the adsorbed state; these configurations are presented in Figure 2. In contrast with experimental observations, the adsorption behavior of the

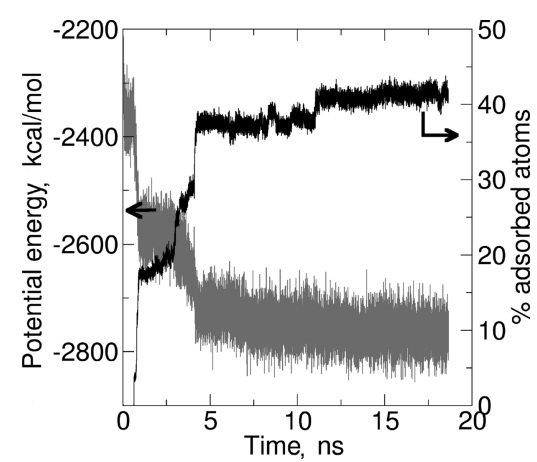

Figure 1. Correlation of the fraction of adsorbed atoms with potential energy in vacuum simulations. The system shown is $6 \mathrm{NGBP}$ on gold at $310 \mathrm{~K}$. Similar results are obtained for other systems.

three peptides is similar. All three adsorb extensively on gold, as illustrated in Figure 3, with the fraction of adsorbed amino acids varying by less than $10 \%$. To quantify the extent of binding, we determine the potential energy of adsorption, $\Delta U$, by taking the difference in potential energies of adsorbed and free peptides per amino acid. By this measure, the non-goldbinding peptide $[-13 \mathrm{kcal} / \mathrm{mol} / \mathrm{AA}]$ binds more strongly than the two gold-binding peptides $[\Delta U=-10 \mathrm{kcal} / \mathrm{mol} / \mathrm{AA}]$. Our vacuum simulations are not consistent with experimental evidence, suggesting that they are not an appropriate probe of adsorption. This differs from platinum binding peptides, in which case vacuum simulations correlate with experimental observations. ${ }^{13}$ Although the reason for this is unclear, one possibility is the length of the peptides. Those studied in ref 13 had only seven residues, whereas our three-repeat peptides have 42 residues. Another possibility is the strength of interaction with the surface. Water adsorption energies for the platinum surface studied in ref 13 are about three times those on gold; ${ }^{41}$ as a result, the mechanism for adsorption may differ between the two cases.

Simulations of Peptides on Gold with Explicit Water. Based on the results presented above, solvation is required to understand adsorption of peptides on gold surfaces. Because these simulations are more computationally demanding, we assume that the most favorable initial orientations for adsorption in vacuum apply when water is present. We thus do not test 10 different orientations as was done in vacuum but use the most favorable orientation in vacuum as the starting point for simulations of solvated peptides. The simulations were run until the fraction of adsorbed atoms remained constant for at least $10 \mathrm{~ns}$; durations were $30-40 \mathrm{~ns}$, as illustrated in Figure 4. In contrast to the simulations in vacuum, those run with explicit water are consistent with experimental observations. Specifically, $3 \mathrm{GBP}$ and 6GBP adsorb extensively to the surface, whereas 6NGBP adsorbs poorly. It is important to examine this observation. Conventional MD simulations are not able to ensure minimum energy structures. Enhanced sampling techniques such as replica methods could provide more certainty but are not feasible for peptides of this size. It is thus possible that the minimum energy structure for $6 \mathrm{NGBP}$ is more extensively adsorbed but not observed due to computational limitations. To assess reproducibility, we tested the opposite orientation of 6 NGBP relative to the surface. This resulted in similar fractions of adsorbed amino acids, although there was some variation in which amino acids adsorbed. We conclude that, within the limitations of the simulations, behavior corresponding to experiment is observed, although it is possible that this is fortuitous. The remainder of the paper links computationally observed 


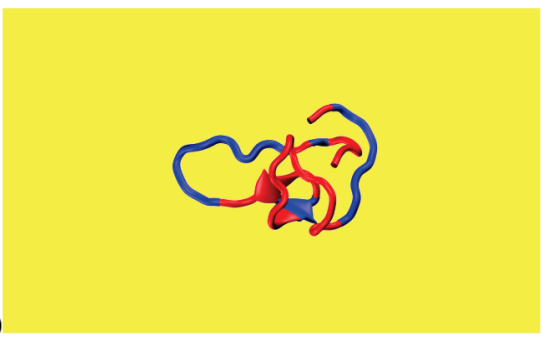

a)
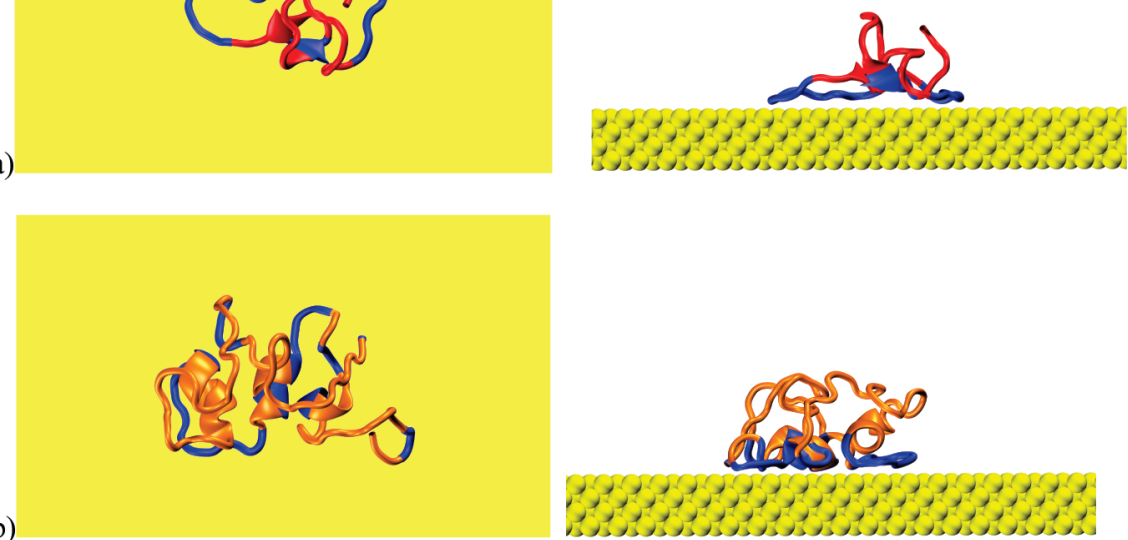

b)
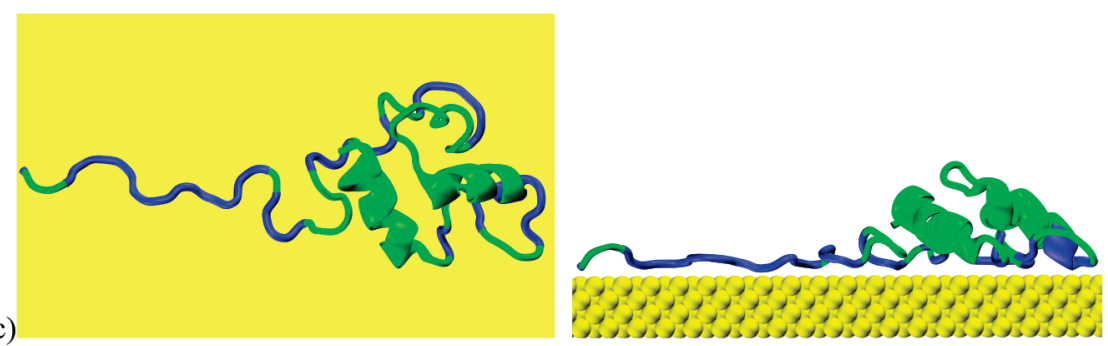

Figure 2. Final adsorbed conformation of (a) 3GBP, (b) 6GBP, and (c) 6NGBP on Au(001) from MD simulations in vacuum at $310 \mathrm{~K}$. The tube represents the backbone of the protein. Adsorbed sections are shown in blue. The texture of the gold surface [in yellow] is omitted from the top views for clarity.

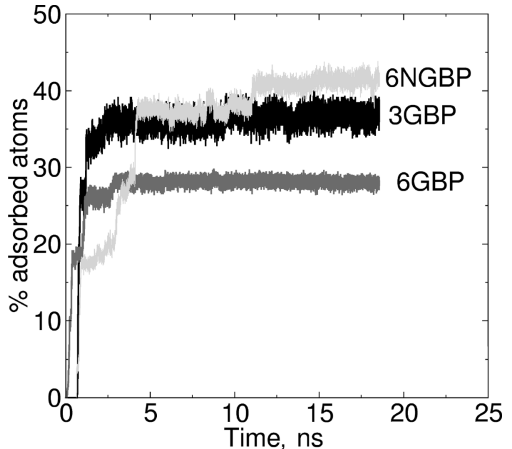

Figure 3. Percentage of adsorbed amino acids from vacuum MD simulations.

solution behavior with adsorption, which is robust despite limitations in comparing with experimental behavior.

The percentage of adsorbed atoms is higher for the goldbinding peptides $[3 \mathrm{GBP}=50 \%, 6 \mathrm{GBP}=33 \%]$ than for the non-gold-binding peptide [ $6 \mathrm{NGBP}=18 \%]$. The main difference between vacuum and solvated simulations is the behavior of 6NGBP: it adsorbs to a far lesser extent [18 vs 40\%] over a period twice as long. In contrast, vacuum and solvated simulations result in similar fractions of adsorbed atoms for $3 \mathrm{GBP}$ and 6GBP. At about $30 \mathrm{~ns}$, the shorter gold-binding peptide alters its configuration to increase its contact with the surface, something that was not observed in vacuum. This alteration may be a function of its smaller size or of the structural motif [primarily random coil].

The final adsorbed conformations are presented in Figure 5, from which it is apparent that after adsorption a substantial portion of $6 \mathrm{NGBP}$ does not interact with the surface. Also provided in the figure are the initial configurations. Interaction

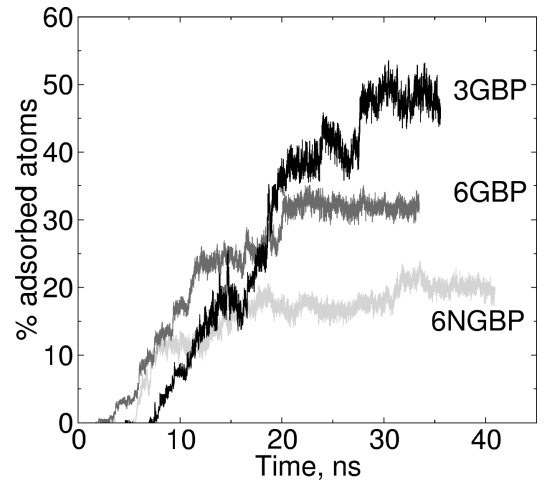

Figure 4. Percentage of atoms adsorbed on $\mathrm{Au}(001)$ as a function of time from explicit water simulations. The first nonzero data point represents the time at which the first atom adsorbs, as defined by being within $5 \AA$ of the gold surface.

with the surface alters the configuration of the gold-binding peptides, whereas the configuration of the non-gold-binding peptide changes little with adsorption. This is confirmed by comparison of the RMSD between adsorbed and initial conformations: $10 \AA$ for $6 \mathrm{GBP}, 7 \AA$ for $3 \mathrm{GBP}$, and $2 \AA$ for 6 NGBP.

With the aid of Table 3 , we ask if the amino acids that adsorb to the gold surface are coincident with those isolated in homopolypeptide experiments. ${ }^{11}$ This table overlays the group of amino acids identified experimentally as gold binding in homopolypeptides, with those we observe to bind to gold in mixed sequences. It is striking that the ability of an individual amino acid to interact with the gold surface appears to have little to do with its binding behavior in our simulations. The non-gold-binding peptide contains a stretch of four gold-binding amino acids [IRRD], which does not appear to drive interactions 


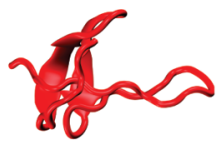

a)
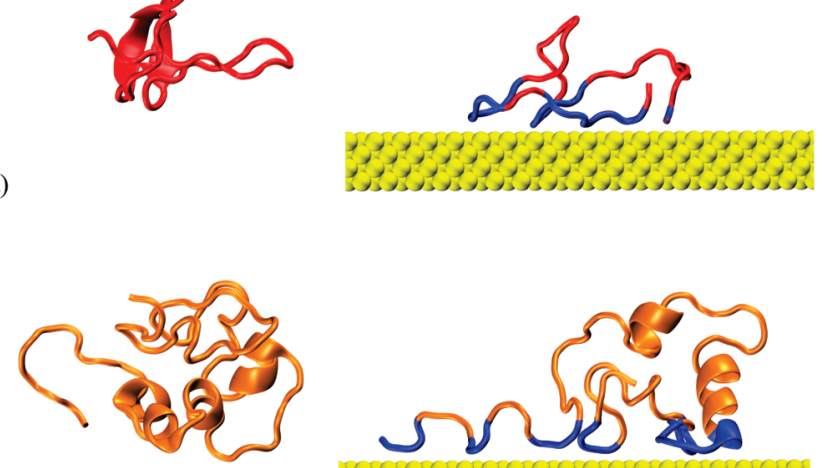

b)

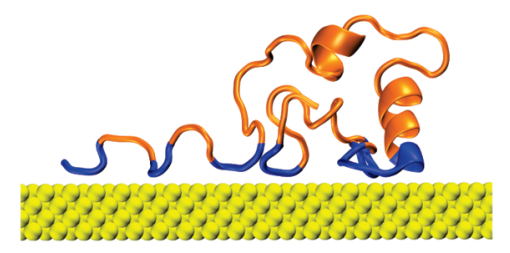

c)
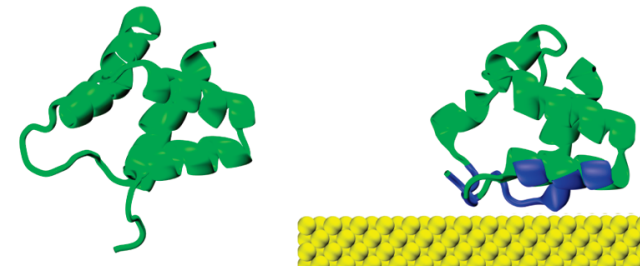

Figure 5. Initial and final adsorbed conformation of (a) 3GBP, (b) $6 \mathrm{GBP}$, and (c) 6NGBP on $\mathrm{Au}(001)$ from explicit water simulations. Initial conformations: left side. Adsorbed configurations: right side. Images do not show the entire simulation box to facilitate display of the protein. The protein backbone is shown as a tube or ribbon; portions colored dark blue are adsorbed. Water molecules are omitted for clarity.

Table 3. Adsorption Behavior of the Six and Three Repeats in 6 NGBP, 6GBP, and 3GBP on Au(001) from Explicit Water Simulations $^{a}$

\begin{tabular}{|c|c|}
\hline peptide & peptide sequence \\
\hline 6NGBP & 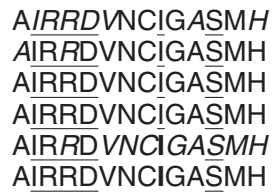 \\
\hline 6GBP & 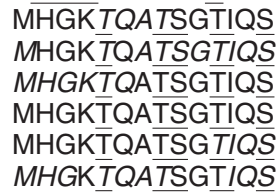 \\
\hline 3GBP & 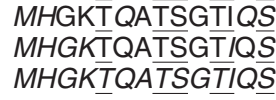 \\
\hline
\end{tabular}

${ }^{a}$ Adsorbed amino acids are italicized and amino acids that target gold experimentally are underlined. ${ }^{11}$

with the surface. The gold-binding peptide contains a stretch of four amino acids that were reported as nonbinding [MHGK]: in the shorter $3 \mathrm{GBP}$, this sequence is adsorbed in all three repeats, although it does not appear to drive binding for 6GBP.

We conclude that simulations of the peptides in explicit water provide a reasonable description of an intermediate phase in adsorption to gold surfaces. They cannot provide information about the early stages where peptides change orientation relative to the surface, nor can they ensure an equilibrium conformation of the peptide at the surface. With these limitations in mind, it appears that amino acid identity and associated characteristics is not a driving force for adsorption in this system. Amino acid sequence may be important, but this remains unclear. We now investigate the correlation of peptide flexibility and stability with adsorption as assessed from our simulations. Stability is the degree to which a peptide retains a given configuration: not only secondary structure, but also smaller changes that precede changing secondary structure, such as changes in backbone configuration. Within a given configuration, each atom is free to explore a local area, similar in spirit to the B factors obtained in crystallography. We associate this local area with the idea of flexibility: atoms able to explore larger local areas are more flexible than those whose motion is constrained to small regions.

We first ask if conformational stability plays a role in the differing adsorption of NGBP and GBP to gold. To assess this, we consider two quantities: the radius of gyration of the peptide over the course of the adsorption simulation and changes in backbone configuration between the initial and adsorbed states. The former reveals changes in the overall size of the peptide, while the second reveals changes in secondary structure. ${ }^{42}$ The radius of gyration is the root-mean-square distance of atoms from the peptide's center of mass

$$
R_{\mathrm{g}}=\sqrt{\frac{\sum_{i=i}^{N} m_{i}\left(\vec{r}_{i}-\vec{r}_{\mathrm{COM}}\right)^{2}}{\sum_{i=1}^{N} m_{i}}}
$$

where $N$ is the number of atoms, $m_{i}$ and $\vec{r}_{i}$ are the mass and position of atom $i$, and $\vec{r}_{\mathrm{COM}}$ is the center-of-mass position of the peptide. To assess changes in backbone configuration, we compare dihedral angles derived from four consecutive $\alpha$ carbons, between the initial and adsorbed configurations. The initial configuration is averaged over the first 1 ns of our simulations and the adsorbed configuration over the last $1 \mathrm{~ns}$. The choice of a 1 ns average removes local fluctuations: below we choose a shorter duration to observe these fluctuations as a measure of peptide flexibility. The difference for any given dihedral indicates a change in configuration at that location, and taken together, the set of all dihedrals reveals the conformational stability of the peptide. It is clear from Figure 6 that $3 \mathrm{GBP}$ and 6GBP change conformation more extensively than 6NGBP with adsorption. Because the backbone of $6 \mathrm{NGBP}$ did not change configuration enough that amino acids facing the peptide interior became exposed to the gold surface, the amino acids initially facing the surface adsorbed. In contrast, the conformational changes of the gold-binding peptides allowed a wider range of amino acids to interact with the surface.

We can conclude that either conformational changes enable adsorption, or adsorption leads to conformational changes. In the former case, conformational change occurs prior to adsorption, whereas in the latter it occurs after. Comparison of the time dependence of peptide size with the time over which adsorption occurs, presented in Figure 7, reveals that both occur. The gold-binding proteins change size both prior to and during adsorption. In the case of 6GBP, a large change in size is evident before adsorption occurs, suggesting that this change enabled the adsorption process. The smaller peptide $3 \mathrm{GBP}$ also changes size before adsorption, and continues to do so throughout the adsorption process. In particular, the change in size between 25 and $30 \mathrm{~ns}$ is coincident with a jump in the number of adsorbed atoms [see Figure 4]. In contrast to the gold-binding peptides, the size of 6NGBP remains constant throughout the adsorption process, consistent with the larger configurational stability described above.

Simulations of Peptides in Solution. Given that the stability of the peptides before adsorption appears to play a role, we 

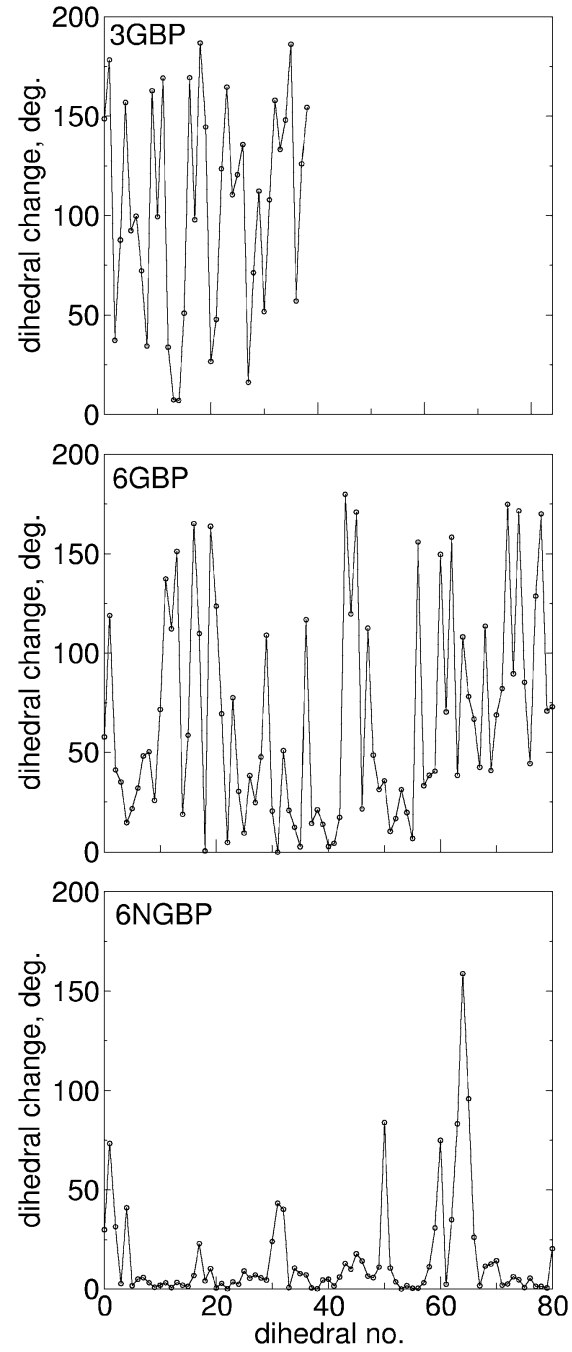

Figure 6. Changes in $\alpha$-carbon dihedral angles with adsorption at $310 \mathrm{~K}$ for $3 \mathrm{GBP}, 6 \mathrm{GBP}$, and 6 NGBP on Au(001). The change is calculated as the difference between the average dihedral in the first and the last nanoseconds in each simulation. Dihedral $n$ corresponds to the dihedral formed by alpha carbons $n, n+1$, $n+2$, and $n+3$.

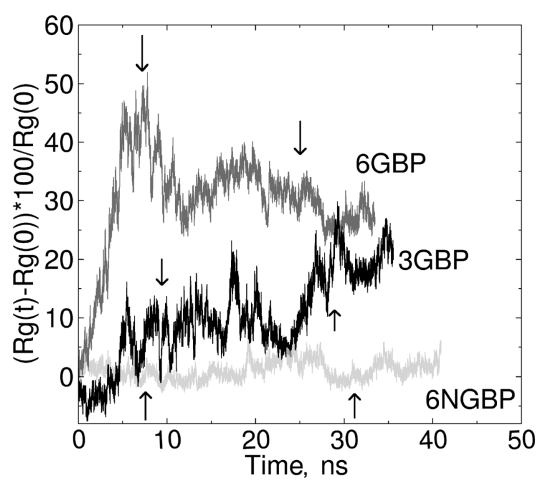

Figure 7. Percent change in radius of gyration of $3 \mathrm{GBP}, 6 \mathrm{GBP}$ and 6 NGBP during adsorption simulations on $\mathrm{Au}(001)$ at $310 \mathrm{~K}$. The arrows in each line indicate the start (defined as the instant when $5 \%$ of peptide's atoms are within $5 \AA$ of the top gold atoms) and the finish (defined as the time after which the percentage of atoms on the surface remains constant) of adsorption.

now examine properties of the peptides before they are influenced by the surface. We investigate both the stability of the backbone configuration, as was done above, and the amplitude of oscillations about the stable configuration, which

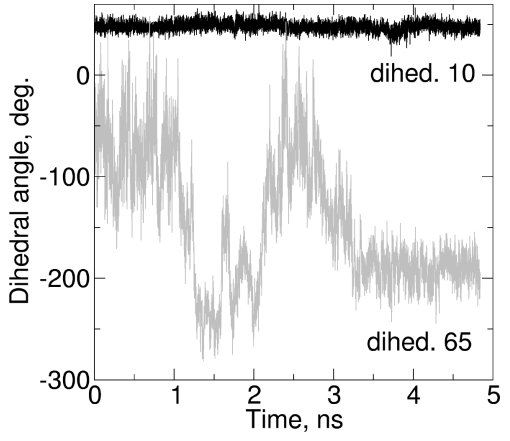

Figure 8. Temporal evolution of two characteristic $\alpha$-carbon dihedral angles for the 6NGBP in solution at $310 \mathrm{~K}$. Dihedral $n$ corresponds to the dihedral formed by alpha carbons $n, n+1, n+2$, and $n+3$. Dihedral changes at nanosecond time-scales probe stability, whereas at picosecond time-scales they probe flexibility.

we term flexibility. To investigate stability and flexibility in solution, we use an approach similar to that described above for adsorption simulations. The approach is based on the dihedral angles formed by four consecutive $\alpha$-carbons. Whereas above we considered the difference between $1 \mathrm{~ns}$ averages of the angle between the initial and final stages of adsorption, here we consider the entire duration of the simulation, asking instead about the change which occurs over time intervals $\tau$ for each dihedral. To place the magnitude of the change in context, we consider the function

$$
C_{\mathrm{dhd}}(\tau)=\left\langle\left|\phi_{t+\tau}-\phi_{t}\right|\right\rangle
$$

where the average is over all possible time origins $t$. Because this function varies between 0 and $180^{\circ}$, we can associate the limit of no change with 0 , and that of maximum possible change as $180^{\circ}$. If the time interval $\tau$ is large, this function probes stability, but if it is small, oscillations of dihedrals about their stable values are monitored, characterizing flexibility. To assign the values of $\tau$ required to monitor stability and flexibility, we consider the decay of the torsional autocorrelation function averaged over all dihedral angles and multiple time origins. The rate of decay varies widely between dihedrals, with an average decay time of $2 \mathrm{~ns}$. We selected a slightly longer time [2.5 ns] as the time scale over which to monitor stability. The choice is illustrated in Figure 8, with one stable [dihedral 10] and one unstable [dihedral 65] dihedral. The figure confirms that conformational changes in the unstable dihedrals are consistent with the chosen interval. Flexibility refers to fluctuations within each stable state, which occur over much shorter time scales: we choose $\tau=10 \mathrm{ps}$ for this purpose. Note that fluctuations are larger in the unstable dihedral, providing the first suggestion that structural stability and flexibility are related.

We first investigate stability by monitoring $C_{\mathrm{dhd}}(\tau=2.5 \mathrm{~ns})$ as a function of dihedral number for peptides in solution and in the adsorbed state described above. From the results, presented in Figure 9, it is immediately clear that the gold-binding peptides are less stable in solution than 6NGBP. The difference occurs not in the size of the conformational change [in fact the largest single change is observed for 6NGBP] but in the number of dihedrals that rotate compared to those that do not. In 6NGBP, most dihedrals do not rotate, resulting in long stretches of immobile dihedrals. In 6GBP, most dihedrals rotate over 2.5 $\mathrm{ns}$, and those that do not are isolated, rather than organized in long immobile stretches. The shorter gold-binding peptide, $3 \mathrm{GBP}$, is intermediate. It is not as stable as $6 \mathrm{NGBP}$, but more 

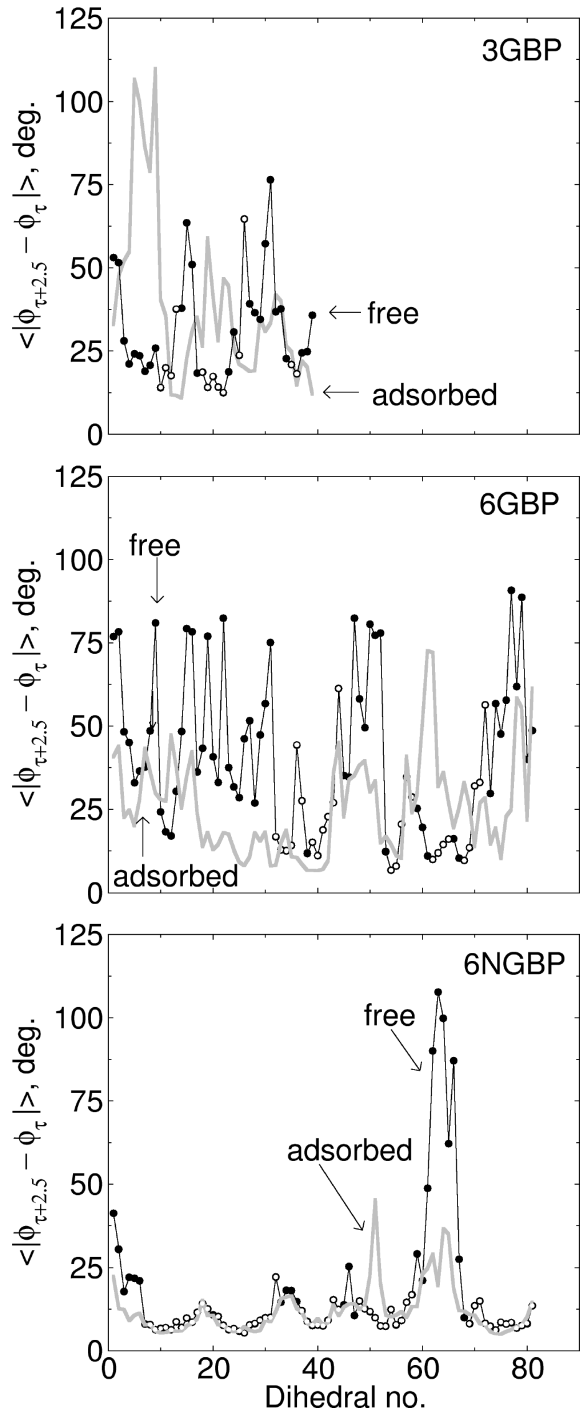

Figure 9. Average change in the $\alpha$-carbon dihedral angles $\left(C_{\text {dhd }}(\tau=\right.$ $\left.2.5 \mathrm{~ns})=\left\langle\left|\phi_{\mathrm{t}+2.5}-\phi_{\mathrm{t}}\right|\right\rangle\right)$ for a $2.5 \mathrm{~ns}$ interval, from simulations of the free (thin lines) and adsorbed (thick lines) peptides in solution. Dihedral $n$ corresponds to the dihedral formed by $\alpha$-carbons $n, n+$ $1, n+2$, and $n+3$. The open symbols correspond to dihedrals participating in secondary structural motifs like helices and sheets in the free molecule.

stable than 6GBP. It is not surprising that the immobile stretches correspond to defined secondary structures such as helices and sheets. Many of the amino acids in 6NGBP are found in helices, and 3GBP has two $\beta$-sheets. As illustrated in Figure 9, these secondary structures have higher stability than other regions of the peptides.

It is also clear from Figure 9 that the stability of free and adsorbed peptide conformations differ. The gold-binding peptide 6GBP becomes significantly more stable on adsorption, with the exception of a small stretch between amino acids 60 and 65 that include a denatured helix. The stability of the shorter gold-binding peptide $3 \mathrm{GBP}$ changes with adsorption, but rather than the adsorbed state being more stable, regions of the peptide with conformational changes are different in the two cases. Amino acids 5-12 and 18-26 lose stability with adsorption, consistent with the disappearance of the $\beta$-sheet to which they belonged.

To investigate peptide flexibility, we monitor two quantities: the oscillation of dihedral angles within a given conformation, $C_{\mathrm{dhd}}(\tau=0.01 \mathrm{~ns})$, and the size of the region explored by each
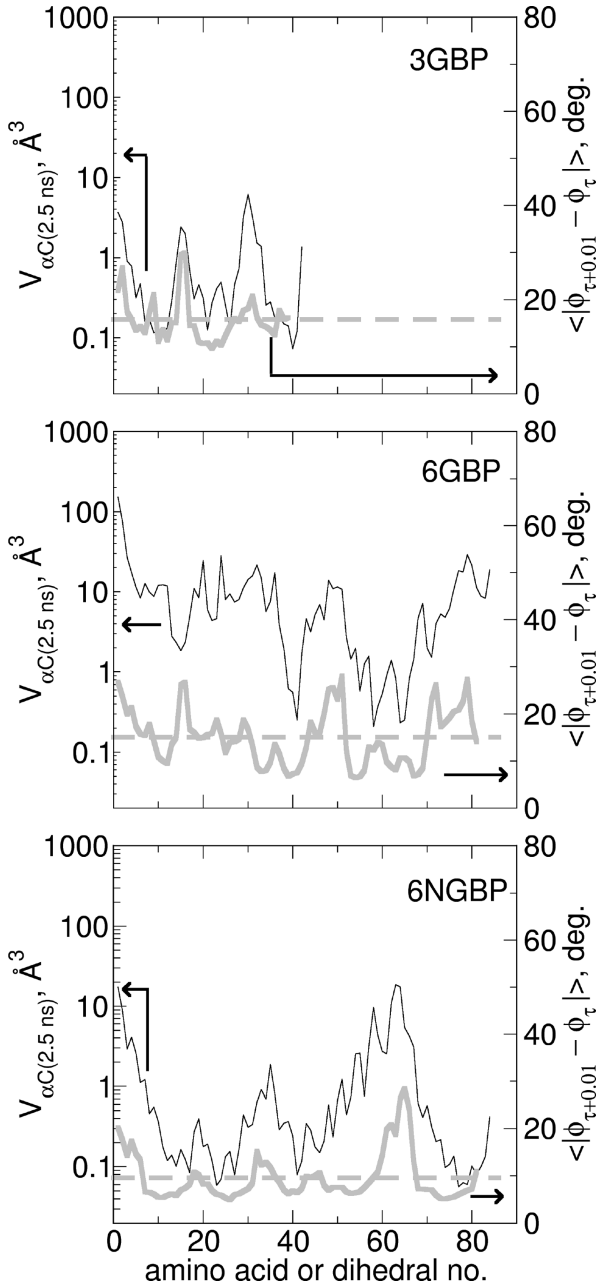

Figure 10. Comparison of peptide flexibility for 3GBP, 6GBP, and 6 NGBP peptides in solution. Volume probed by each $\alpha$-carbon over $2.5 \mathrm{~ns}\left[V_{\alpha \mathrm{c}}\right]$ : thin lines. Change in the $\alpha$-carbon dihedral angles $\left[C_{\text {dnd }}(\tau=0.01 \mathrm{~ns})=\left\langle\left|\phi_{t+0.01}-\phi_{\mathrm{t}}\right|>\right]\right.$ : thick lines. Average change in the $\alpha$-carbon dihedral angles: dashed lines. The $x$-axis represents the amino acid number [for $V_{\alpha \mathrm{c}}$ ] or the dihedral number [for $C_{\text {dhd }}(\tau=$ $0.01 \mathrm{~ns})]$. Dihedral $n$ corresponds to the dihedral formed by alpha carbons $n, n+1, n+2$, and $n+3$.

$\alpha$-carbon, $V_{\alpha \mathrm{CC}}$. We estimate the size of this region with the product of $X, Y$, and $Z$ average displacements of each $\alpha$-carbon, after removing rotation and translation of the peptide as a whole. To do so, we must choose an appropriate time over which to take the displacements. It might seem that the time over which we monitor the dihedral angle $[0.01 \mathrm{~ns}]$ is the correct choice. However, our objective is to identify the volume explored by each $\alpha$-carbon while in a given conformation. For this, we need to take the time interval as equal to the average time between conformational jumps, identified above as $2.5 \mathrm{~ns}$. During this time, many oscillations [time scale of $0.01 \mathrm{~ns}$ ] will occur. The $X, Y$, and $Z$ displacements represent the average size of these oscillations: if we take a time scale of $0.01 \mathrm{~ns}$ we see only one oscillation, which may or may not represent this average. The calculation of $V_{\alpha \mathrm{C}}$ resembles that of the protein RMSD, where a dependence on the number of amino acids has been demonstrated. ${ }^{43}$ We thus expect that $V_{\alpha \mathrm{C}}$ for the six repeat peptides will exceed that of the three repeat peptide, with direct numerical comparison only meaningful between $6 \mathrm{NGBP}$ and 6GBP.

The two measures show similar trends, as illustrated in Figure 10. Regions with large $V_{\alpha \mathrm{C}}$ and high values of $C_{\mathrm{dhd}}(\tau=0.01$ 
ns) coincide, indicating that large flexibility is revealed by both quantities. For the remainder of the discussion, we focus on the volume explored to characterize flexibility. Although both functions provide similar information, this is more physically intuitive.

Variations in flexibility are decoupled from conformational transitions. This is evident by comparing the volumes explored by the 6 NGBP $\alpha$-carbons in amino acids $10-50$ presented in Figure 10 with the changes for 6NGBP dihedrals 10-50 shown in Figure 9 [the dihedral number is approximately equal to the amino acid number]. Within this stretch where the dihedral angles change very little, the volumes explored by those $\alpha$-carbons vary by over an order of magnitude. Comparing $6 \mathrm{GBP}$ and $6 \mathrm{NGBP}$, it is clear that flexibility is higher for the gold-binding peptides. Although both explore volumes with sizes spanning 2 orders of magnitude, for $6 \mathrm{GBP}$ the range $[1-100$ $\AA^{3}$ ] is an order of magnitude greater than that for 6 NGBP $\left[0.1-10 \AA^{3}\right]$. Not only is 6NGBP more stable, the movement of its atoms within a given configuration is more restricted. This is not surprising because $6 \mathrm{NGBP}$ is more structured than $6 \mathrm{GBP}$, with a larger fraction of its atoms in secondary structural motifs. This appears to be important for binding to a surface. The larger flexibility and less stable structure of the gold-binding peptide allow it to more easily search for conformations that can form and hold surface contacts. Interestingly, cross-correlation in flexibility is not required to promote conformational change. Although the extent of individual $\alpha$-carbon flexibility correlates with conformational change, cross-correlations are similar in both peptides. We cannot compare numerical values of the volume explored by 3 GBP to the six repeat peptides; we can only compare the oscillations in dihedral angles. Torsional oscillations in all three peptides range from $10-30^{\circ}$, and as discussed above, those amino acids with large torsional oscillations also explore large volumes. Whereas the absolute values of volumes explored by the gold-binding peptide clearly exceed those of the non-gold-binding peptide, with torsional oscillations the difference is in the number of amino acids with large values of $C_{\text {dhd }}(\tau=0.01 \mathrm{~ns})$. We illustrate this by the dashed lines, which indicate the average over all amino acids: for both goldbinding peptides this is $15^{\circ}$, whereas for the non-gold-binding peptide, the average is only $10^{\circ}$.

In Figure 11 we compare the flexibility of the peptides in solution and adsorbed to the surface: the latter is determined by averaging over the last $5 \mathrm{~ns}$ of adsorption simulations. Comparing the two curves, it is evident that binding reduces flexibility in the regions where the backbone conformations are changing in solution. This is likely a result of interactions with the surface reducing conformational changes. In general, flexibility in stable regions [i.e., dihedrals with changes close to zero in Figure 9] is not altered by adsorption. Examples are dihedrals in the 50-70 range for 6GBP and the 10-50 range for 6NGBP: these regions are more stable because most of the amino acids are in helices.

The flexibility of these regions is greater for the gold-binding peptides [see Figure 10], which may aid in furthering adsorption once surface contact is established. The overall flexibility of $3 \mathrm{GBP}$ is not altered by its interaction with the surface even though the location of flexible and less flexible regions changes: for example, adsorption increases flexibility of amino acids 5-12 and 18-26 while decreasing that of other regions. We also note that the peptide completely denatures during adsorption. We highlight the flexibility of the QAT motif in 3GBP and 6GBP because NMR and circular dichroism experiments indicate that this motif interacts with gold ions and is thus critical
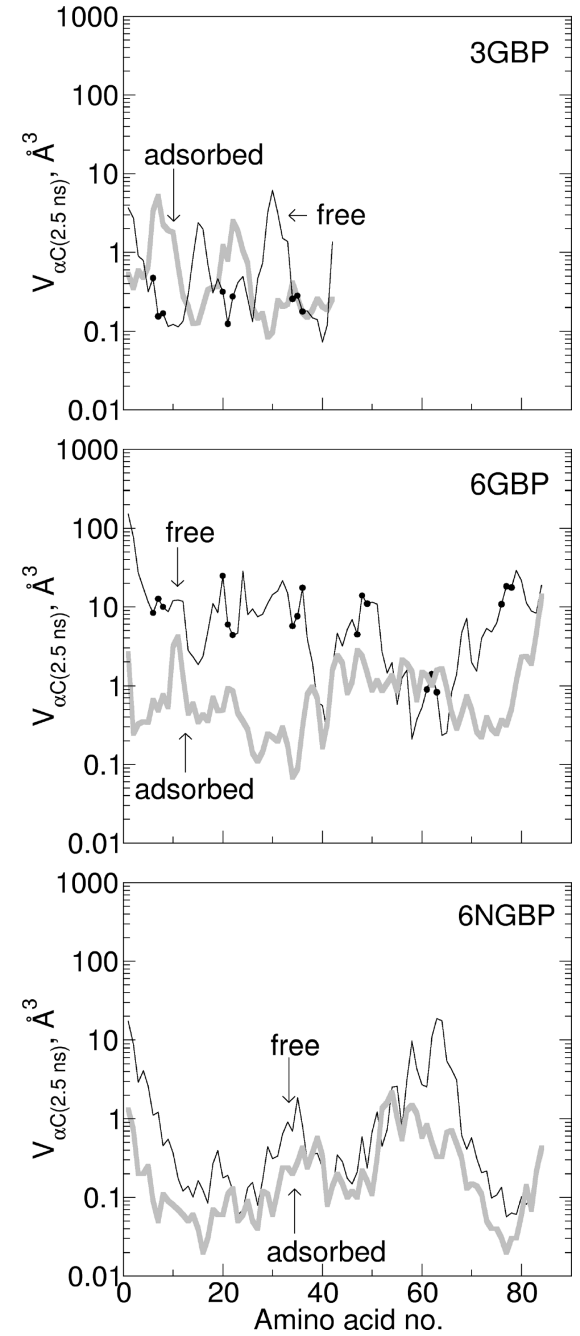

Figure 11. Average volume probed by each $\alpha$-carbon for 3GBP, $6 \mathrm{GBP}$, and 6 NGBP for a $2.5 \mathrm{~ns}$ interval $\left(V_{\alpha \mathrm{C}}(2.5 \mathrm{~ns})\right)$ at $310 \mathrm{~K}$. The thin lines are from $5 \mathrm{~ns}$ long simulations of the free peptide in solution; the thick gray lines are from the final 5 ns of adsorption simulations. The black dots correspond to the QAT motif that exists only in the gold-binding peptides and is relevant for precipitation of gold from solution.

for the peptides' ability to precipitate gold from solution. ${ }^{17}$ This motif is not significantly more flexible than the remainder of the peptide, indicating that although its interactions with the surface are important, they are enabled by flexibility of the peptide as a whole rather than being more flexible than average themselves.

We summarize our results on flexibility and stability in pictorial form in Figure 12. The left-hand side of images indicates flexibility and the right-hand side row stability of the peptides in solution. We choose the dihedral angle oscillations to represent flexibility because absolute values of $V_{\alpha \mathrm{C}}$ for three and six repeat peptides cannot be compared. For flexibility, the color scale goes from blue [less flexible] to red [more flexible]. We reverse the scale for stability [blue $=$ more stable, red $=$ less stable] so that quantities enabling binding [high flexibility, low stability] are both red. We first note a reasonable correlation between the two quantities: high flexibility occurs in the least stable regions. This is not surprising since local motions enable the conformational transitions that decrease stability. Within each peptide, the more stable and least flexible regions are those with defined secondary structures. This is also not surprising, since the defined geometry and intramolecular hydrogen bonding 
a)

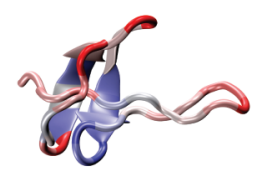

b)

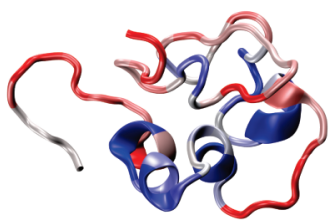

c)

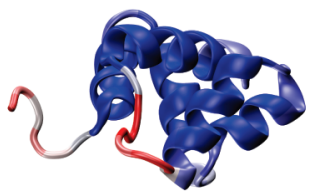

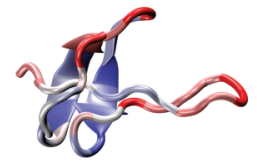
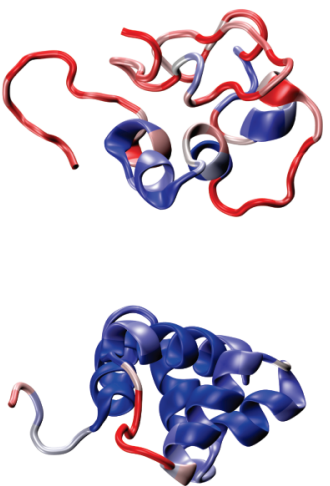

Figure 12. Summary of peptide flexibility and stability in solution for (a) 3GBP, (b) 6GBP, and (c) 6NGBP. The left-hand side illustrates flexibility and is based on $C_{\text {dhd }}(\tau=0.01 \mathrm{~ns})$, as presented in this figure. The right-hand side illustrates stability and is based on $C_{\text {dhd }}(\tau=2.5$ ns), as presented in Figure 10. The color scale correlates with the values for $C_{\text {dhd }}$, with blue indicating low flexibility and high stability and red indicating high flexibility and low stability.

in these regions will naturally limit conformational exploration. Within these categories, the gold-binding peptides are both more flexible and less stable. For example, regions with defined secondary structure in the non-gold-binding peptide are closer to the blue end of the scale than in the gold-binding peptides. A similar observation holds for regions that are random coil: in the gold-binding peptides, these are closer to the red end of the scale than in the non-gold-binding peptide. As presented above, the solution structures for the peptides were not available and were predicted using structure predictors. While we are confident that the structures of the gold-binding peptides are reasonable, we have no such confirmation for the non-goldbinding peptide. The fact that mobility varies between the two even in regions of similar secondary structure makes this less problematic: even if 6NGBP were less structured than predicted, the nonstructured regions may remain less flexible than the goldbinding peptides.

Because the two classes of peptides in our study have the same number of amino acids that interact strongly with gold, it is reasonable to conclude that the higher flexibility and lower conformational stability of the gold-binding peptides enables adsorption. The gold-binding peptides have high affinity for gold both because they contain amino acids that interact strongly with gold and because their lower stability and high flexibility enable them to establish multiple contacts with the gold surface. This is not always the case: in our vacuum simulations, both types of peptides adsorbed extensively and are stable in vacuum.

Thus, in the absence of water, high flexibility and low stability are not a requirement for adsorption to gold. This is not surprising because interactions between protein and gold are stronger than those between protein and protein, as estimated by comparing the van der Waals parameters for all the atoms. Simulations of adsorption of protein-like chains on generic surfaces indicate that when protein-surface interactions are strong, even highly stable proteins [such as our peptides in vacuum] can adsorb strongly. ${ }^{19}$ In those conditions the free energy of adsorption is dominated by the enthalpy of adsorption

[or, at constant volume, by the energy of adsorption], because the protein-surface interaction is highly negative (e.g., the adsorption energy for $3 \mathrm{GBP}$ in vacuum is $\Delta U_{\mathrm{GBP} \text {,vacuum }}=-450$ $\mathrm{kcal} / \mathrm{mol}$ ). In this case, the loss of conformational entropy on adsorption is easily overcome. When the peptides are solvated, the enthalpy of adsorption includes the interactions of water with protein and gold. The favorable interactions of the protein with the gold surface are thus balanced by the cost of displacing water-protein and water-gold interactions, with the result that the enthalpy of adsorption is much less favorable than in vacuum (e.g., $\Delta U_{3 \mathrm{GBP} \text {,solvated }}=-50 \mathrm{kcal} / \mathrm{mol}$ ). As a result, the entropic cost of adsorption is more important. If we imagine the adsorption process to proceed in two steps: displacing water, followed by establishing contact with the surface, the first presents a transition state barrier that is absent in vacuum.

Overcoming this barrier requires sampling a larger range of conformations before finding one that provides favorable interactions with the surface. High flexibility and low stability allow for this larger sampling and are thus important when the systems are solvated.

As mentioned above, several amino acids that adsorb in our simulations do not belong to the gold-binding group as defined by others. ${ }^{11}$ Given the importance of peptide flexibility in adsorption to gold, it is useful to consider these results. Willett's experiments showed that homopolypeptides made of Thr, Ser, Ile, Arg, Asp, and Pro adsorb on gold, so we can infer that those individual amino acids have affinity for gold. However, we should not necessarily conclude that the other amino acids tested do not have affinity for gold. It is possible that those homopolypeptides do not adsorb because properties of the homopolypeptide as a whole (stability and flexibility of its solution structure) do not allow it, despite high affinity.

\section{Conclusions}

We observe adsorption behavior of the peptides 3GBP, 6GBP, and 6 NGBP on gold surfaces. Within computational limits, this behavior correlates with experimental observations: $3 \mathrm{GBP}$ and 6 GBP adsorb strongly, whereas 6 NGBP does not. ${ }^{5,20}$ This occurs even though all peptides contain the same fraction of amino acids that interact favorably with gold. ${ }^{11}$ Our results indicate that, when the peptides are solvated, strong adsorption on gold takes place only if the peptides have high conformational flexibility and low conformational stability. In contrast, adsorption from vacuum occurs in all cases, despite low flexibility and high stability. This highlights the role of the solvent in adsorption, possibly via its mediation of peptide/surface interactions. The limitations of our approach include the lack of structural data with which to obtain solution structures of the peptides, the period of time over which behavior is monitored, and the nature of interactions with the gold surface. Few studies of peptides interacting with surfaces have appeared due to computational limitations and the difficulty in combining realistic surface representations with the classical potentials used to simulate biological molecules. Our study provides a first approach in which the surface does not react to the surrounding water and peptide and only interacts via dispersive interactions. In this case, effective adsorption at the surface requires displacement of water molecules, which is facilitated if the protein is flexible enough to easily undergo conformational changes. While it is possible that a more realistic surface representation might alter this result, it reflects the relative interactions of water and peptide with the gold. Thus, we expect differences only if the neglected physics differ substantially 
between gold-water and gold-peptide. We note that additional studies at a more detailed level are needed to address those issues that ours cannot: for example the binding of various amino acids to gold surfaces, and the nature of the water and peptide layering near gold.

We conclude by suggesting a new selection tool for designing peptide libraries for cell-display and phage-display protocols. As previously shown, adsorption of peptides on gold and other noble metals like platinum is enhanced by enriching the peptide in amino acids with higher affinity for the metal. ${ }^{11}$ In the case of small peptides on gold, selecting for high peptide flexibility is also required. Thus, identification of new artificial amino acid sequences that adsorb strongly and specifically to particular metals will benefit by selecting for high flexibility.

Acknowledgment. A.V.V. acknowledges the Portuguese National Science Foundation for grant SFRH/BPD/20555/2004/ 0GVL. We thank R. Kramer Campen, S. Abeln, K. Schulten, and his group (A. Aksimentiev, E. Cruz-Chu, P. Freddolino, M. Sotomayor, E. Villa) for helpful discussions in the course of this work.

Supporting Information Available. Parameterization procedure for gold. This material is available free of charge via the Internet at http://pubs.acs.org.

\section{References and Notes}

(1) Ji, B. H.; Gao, H. J. J. Mech. Phys. Solids 2004, 52 (9), 19631990.

(2) Yao, N.; Epstein, A.; Akey, A. J. Mater. Res. 2006, 21 (8), 19391946.

(3) Kroger, N.; Deutzmann, R.; Sumper, M. Science 1999, 286 (5442), $1129-1132$.

(4) Slocik, J. M.; Stone, M. O.; Naik, R. R. Small 2005, 1 (11), 10481052.

(5) Brown, S. Nat. Biotechnol. 1997, 15 (3), 269-272.

(6) Sano, K. I.; Sasaki, H.; Shiba, K. Langmuir 2005, 21 (7), 30903095 .

(7) Lee, S.-K.; Yun, D. S.; Belcher, A. M. Biomacromolecules 2006, 7, $14-17$.

(8) Kramer, R. M.; Li, C.; Carter, D. C.; Stone, M. O.; Naik, R. R. J. Am. Chem. Soc. 2004, 126 (41), 13282-13286.

(9) Whaley, S. R.; English, D. S.; Hu, E. L.; Barbara, P. F.; Belcher, A. M. Nature 2000, 405 (6787), 665-668.

(10) Flynn, C. E.; Mao, C. B.; Hayhurst, A.; Williams, J. L.; Georgiou, G.; Iverson, B.; Belcher, A. M. J. Mater. Chem. 2003, 13 (10), 24142421.

(11) Willett, R. L.; Baldwin, K. W.; West, K. W.; Pfeiffer, L. N. Proc. Natl. Acad. Sci. U.S.A. 2005, 102 (22), 7817-7822.

(12) Braun, R.; Sarikaya, M.; Schulten, K. J. Biomater. Sci., Polym. Ed. 2002, 13 (7), 747-757.

(13) Oren, E. E.; Tamerler, C.; Sarikaya, M. Nano Lett. 2005, 5 (3), 415419.

(14) Tsai, A. M.; Udovic, T. J.; Neumann, D. A. Biophys. J. 2001, 81 (4), 2339-2343.

(15) Siddiqui, K. S.; Cavicchioli, R. Annu. Rev. Biochem. 2006, 75, 403433.
(16) Tang, K. E. S.; Dill, K. A. J. Biomol. Struct. Dyn. 1998, 16 (2), 397411.

(17) Kulp, J. L.; Sarikaya, M.; Evans, J. S. J. Mater. Chem. 2004, 14 (14), $2325-2332$

(18) Kantarci, N.; Tamerler, C.; Sarikaya, M.; Haliloglu, T.; Doruker, P. Polymer 2005, 46 (12), 4307-4313.

(19) Liu, S. M.; Haynes, C. A. J. Colloid Interface Sci. 2005, 284 (1), $7-13$.

(20) Tamerler, C.; Duman, M.; Oren, E. E.; Gungormus, M.; Xiong, X. R.; Kacar, T.; Parviz, B. A.; Sarikaya, M. Small 2006, 2 (11), 1372-1378.

(21) Tamerler, C.; Oren, E. E.; Duman, M.; Venkatasubramanian, E.; Sarikaya, M. Langmuir 2006, 22 (18), 7712-7718.

(22) MacKerell, A. D., Jr.; Bashford, D.; Bellott, M.; Dunbrack, R. L., Jr.; Evanseck, J. D.; Field, M. J.; Fischer, S.; Gao, J.; Guo, H.; Ha, S.; Joseph-McCarthy, D.; Kuchnir, L.; Kuczera, K.; Lau, F. T. K.; Mattos, C.; Michnick, S.; Ngo, T.; Nguyen, D. T.; Prodhom, B.; Reiher III, W. E.; Roux, B.; Schlenkrich, M.; Smith, J. C.; Stote, R.; Straub, J.; Watanabe, M.; Wiorkiewicz-Kuczera, J.; Yin, D.; Karplus, M. J. Phys. Chem. B 1998, 102, 3586-3616.

(23) Neria, E.; Stefan, F.; Martin, K. J. Chem. Phys. 1996, 105 (5), 19021921.

(24) Casalone, G.; Tantardini, G. F. J. Chem. Soc., Faraday Trans. 1990, 86 (22), 3793-3795.

(25) Michaelides, A. Appl. Phys. A 2006, 85 (4), 415-425.

(26) Teobaldi, G.; Zerbetto, F. J. Phys. Chem. C 2007, 111 (37), 1387913885.

(27) Kohlmeyer, A.; Witschel, W.; Spohr, E. Chem. Phys. 1996, 213 $(1-3), 211-216$.

(28) Neves, R. S.; Motheo, A. J.; Fartaria, R. P. S.; Fernandes, F. J. Electroanal. Chem. 2007, 609 (2), 140-146.

(29) Shelley, J. C.; Berard, D. R. Computer simulations of water physisorption at metal-water interfaces. In Reviews in Computational Chemistry; Lipkowitz, K. B., Boyd, D. B., Eds.; Wiley-VCH: New York, 1998; Vol. 12, pp 137-205.

(30) Berard, D. R.; Patey, G. N. J. Chem. Phys. 1992, 97 (6), 4372-4379.

(31) Schravendijk, P.; van der Vegt, N.; Delle Site, L.; Kremer, K. ChemPhysChem 2005, 6 (9), 1866-1871.

(32) Schravendijk, P.; Ghiringhelli, L. M.; Delle Site, L.; van der Vegt, N. F. A. J. Phys. Chem. C 2007, 111 (6), 2631-2642.

(33) Heinz, H.; Vaia, R. A.; Farmer, B. L.; Naik, R. R. J. Phys. Chem. C 2008, 112 (44), 17281-17290.

(34) Phillips, J. C.; Braun, R.; Wang, W.; Gumbart, J.; Tajkhorshid, E.; Villa, E.; Chipot, C.; Skeel, R. D.; Kale, L.; Schulten, K. J. Comput. Chem. 2005, 26 (16), 1781-1802.

(35) Humphrey, W.; Dalke, A.; Schulten, K. J. Mol. Graph. 1996, 14 (1), 33-38.

(36) Chivian, D.; Kim, D. E.; Malmstrom, L.; Schonbrun, J.; Rohl, C. A.; Baker, D. Proteins: Struct., Funct., Bioinf. 2005, 61 (7), 157-166.

(37) Cuff, J. A.; Clamp, M. E.; Siddiqui, A. S.; Finlay, M.; Barton, G. J. Bioinformatics 1998, 14 (10), 892-893.

(38) Kneller, D. G.; Cohen, F. E.; Langridge, R. J. Mol. Biol. 1990, 214 (1), 171-182.

(39) Rost, B.; Yachdav, G.; Liu, J. F. Nucleic Acids Res. 2004, 32, W321W326.

(40) Schroder, C.; Rudas, T.; Boresch, S.; Steinhauser, O. J. Chem. Phys. 2006, 124 (23), 234907.

(41) Meng, S.; Wang, E. G.; Gao, S. W. Phys. Rev. B 2004, 69 (19), 195404.

(42) Prestrelski, S. J.; Williams, A. L., Jr.; Liebman, M. N. Proteins: Struct., Funct, Bioinf. 1992, 14, 430-439.

(43) Carugo, O.; Pongor, S. Protein Sci. 2001, 10, 1470-1473. BM9002464 